\section{Chromatic relationships in metacontrast}

\section{suppression $^{1}$}

WILLIAM BEVAN, JOHN JONIDES, and STANLEY C. COLLYER, The Johns Hopkins University, Baltimore, Md. 21218

Total masking of a colored disk in the Werner disk-ring paradigm was found to be a function of the interaction between its color and the color of the ring that followed it. Suppression occurred most frequently when disk and ring hues were identical, least often when complementary hues were paired, and with intermediate frequency when hues were different but noncomplementary. Results were consistent with predictions from simultaneous color contrast, and support integration theories of metacontrast.

The metacontrast or backward masking paradigm typically involves the rapid sequential presentation of two different visual stimuli whose contours do not spatially overlap. Under the proper conditions of temporal sequencing and with particular stimulus configurations, the second or masking figure will entirely inhibit perception of the first or test figure. This phenomenon, metacontrast suppression in Kahneman's terminology (1968), has been perhaps most frequently studied with the disk-ring display of Werner (1935), where the test figure is a solid disk and the masking figure a ring whose inner diameter is coincident with the contour of the disk. Among the variables studied with this display have been both test and masking stimulus duration and interstimulus interval (e.g., Kolers \& Rosner, 1960), differences in brightness between disk and ring (Schiller \& Smith, 1966; Kolers, 1962), and ring-disk contour separation (Cox, Dember, \& Sherrick, 1969; Kolers \& Rosner, 1960; Werner, 1935).

As Kahneman (1968) points out, a central theme shared by most students of masking is the notion that response processes elicited by sequentially presented forms may overlap temporally, even if the forms themselves do not. There is disagreement, however, as to whether the resulting perception is an integration of the two response processes or whether the second event interrupts the processes associated with the first. The present experiment studies color interactions between lisk and ring in the Werner paradigm. It explores the hypothesis that, if test and masking stimuli are integrated, greater phenomenal distance between the hues of ring and disk will enhance detection of the disk in a fashion predicted from the dynamics of simultaneous color contrast (Graham \& Brown, 1965). METHOD

One male and three female Ss, aged 19-26, were screened for possible color weaknesses with the Ishihari color plates. Each served in two practice and five daily experimental sequences of approximately $11 / 2 \mathrm{~h}$ duration each. Each sequence was divided in half with a rest interval of at least $1 \mathrm{~h}$ intervening between halves. Additional short rest periods were provided upon S's request.

A three-channel Scientific Prototype tachistoscope (Auto Tach, Model GB) was used for the sequential presentation of disk and ring stimuli. The background intensity of the stimulus fields, determined with the aid of a Macbeth Illuminometer, measured $10.7 \mathrm{fL}$. The intensity of the interstimulus field was too low to be measurable with the Macbeth, but just high enough to make possible the fixation of the viewing area between trials.

The outside diameter of the ring subtended a visual angle of $1 \mathrm{deg} 34 \mathrm{~min}$; the ratio of outside to inside diameters was 4:3. Disk diameter was equal to the inside diameter of the ring. Color transparencies of the figures were prepared by placing ring and disk stencils over pieces of Munsell colored paper and photographing them. Four chromatic colors chosen for study were selected at $90-\mathrm{deg}$ intervals around the Munsell color wheel: red (10 RP), yellow (5 Y), green $(10 \mathrm{G})$, and blue (5 PB). Lightness and saturation were uniform, at Munsell Value 6 and Chroma 10. A fifth color, a gray of Value 6, was also chosen.

Sessions were conducted in a darkened room. Approximately $5 \mathrm{~min}$ dark adaptation was allowed in order for $S$ to be able to see the outlines of the fixation field. Each sequence consisted of random presentations of all 25 combinations of disk and ring at each of 10 interstimulus intervals, with ISI defined as the temporal separation between the offset of the disk and the onset of the ring. Disk duration was set at $18 \mathrm{msec}$, and ring duration at $100 \mathrm{msec}$; ISIs ranged from 5 to $68 \mathrm{msec}$, in steps of 7 msec. Time between trials was $5-10 \mathrm{sec}$.

$\mathrm{S}$ was requested to respond after each trial by identifying the color of the disk just presented, regardless of whether it looked like an actual disk or a formless spot of color. If nothing but a white area was seen inside the ring, $S$ responded by saying "No." Catch trials (i.e., ring without disk) were not included, and $S$ was aware of this fact.

\section{RESULTS AND DISCUSSION}

Table 1 indicates the relative frequency with which the disk failed detection, at each ISI, when disk and ring were either (A) identical in hue, (B) of different but not complementary hues, or (C) complementary. Entries in the table indicate the percentage of trials on which $S$ reported seeing nothing inside the ring. It is clear that failure to detect the disk occurred least frequently when its hue was complementary to that of the ring and most frequently when both stimulus figures were identical in hue. The other category, different but noncomplementary hues, yields intermediate levels of masking. A Friedman two-way analysis of variance indicates significant differences among these three conditions at $p<.01$. Wilcoxon tests indicate that Column $\mathrm{C}$ differs from both Columns $A$ and $B$ at $p<.005$, but the difference between (A) and (B) fails significance at the .05 level. Further inspection of the data, however, reveals that differences between the columns do not occur uniformly across all ISIs but relate instead to general level of detectability. Under the conditions of the present experiment, maximal effects occur within an intermediate (12- to $40-\mathrm{msec}$ ) range of ISIs, and Columns $A$ and $B$ differ from each other at the .05 level for this range. In the case of longer and shorter intervals, masking of the disk would appear to have occurred under all conditions either too frequently or too seldom to permit the appearance of differences due to chromatic interactions.

The results of Table 1 can be understood in terms of the effects related to the processes of simultaneous color contrast.

Table 1

Percent Trials on Which $S$ Failed to Detect the Disk, When Disk and Ring Pairs were (A) Identical in Hue, (B) Different But Not Complementary, and (C) Complementary

\begin{tabular}{|c|c|c|c|}
\hline \multirow{2}{*}{$\begin{array}{l}\text { Inter- } \\
\text { stimulus } \\
\text { Interval } \\
\text { (ISI) }\end{array}$} & \multicolumn{3}{|c|}{ Ring-Disk Chromatic Relations } \\
\hline & $\begin{array}{c}\text { (A) } \\
\text { Identical }\end{array}$ & $\begin{array}{c}\text { (B) } \\
\text { Noncomple- } \\
\text { mentary }\end{array}$ & $\begin{array}{c}\text { (C) } \\
\text { Comple- } \\
\text { mentary }\end{array}$ \\
\hline 5 & 66.3 & 70.6 & 63.8 \\
\hline 12 & 63.8 & 53.8 & 37.5 \\
\hline 19 & 35.0 & 37.5 & 23.8 \\
\hline 26 & 31.3 & 22.5 & 16.3 \\
\hline 33 & 15.0 & 11.9 & 11.3 \\
\hline 40 & 8.8 & 7.5 & 2.5 \\
\hline 47 & 2.5 & 3.1 & 1.3 \\
\hline 54 & 1.3 & 1.3 & 0 \\
\hline 61 & 0 & 0 & 0 \\
\hline 68 & 1.3 & 0 & 0 \\
\hline Overall $\overline{\mathrm{X}}$ & 22.5 & 20.8 & 15.6 \\
\hline
\end{tabular}


Fig. 1. Percent trials on which $S$ failed to detect the disk, averaged across all five ring colors. Data for adjacent ISIs were combined: 5 and 12,19 and 26,33 and 40 , 47 and 54, 61 and $68 \mathrm{msec}$.

The conditions of the present experiment are similar in many respects to those producing optimal color contrast. Graham \& Brown (1965, p. 460) describe these conditions as including total enclosure of the test field by the inducing field, minimal separation between their boundaries, equal brightnesses of the two fields, lack of surface texture, etc. With a disk-ring paradigm, presentation of a colored ring may induce perception of the complementary color adjacent to its inner boundary; this complement in turn may interact with the color of the disk just presented, since a clear perception of the disk is still in the process of formation. The result, in the case of a red-green disk-ring pairing, for example, may thus be an enhancement of the red disk and consequently a reduced probability of masking. On the other hand, when disk and ring are identical in hue, the probability of masking should be maximal, since the inner boundary of a red ring, for example, will tend toward a green tinge and therefore desaturate the emerging contour of a red disk. Finally, when ring and disk colors are neither complementary nor identical, the effect should be partial desaturation of the disk contour, producing an intermediate level of masking. Further study is needed to corroborate this explanation and to provide support for predictions of greater precision than the above. In general terms, however, the direction of the results indicates support for integration theories of masking.

Finally, Fig. 1 indicates the likelihood of masking displayed by each of the five disks, averaged across all rings. It may be seen that maximum suppression in all cases occurs at the shortest ISI, indicative of a Type A masking function (Kolers, 1962). As Kolers points out, such a function is to be expected when a marked imbalance exists between test and masking stimulus, with the imbalance favoring the latter. In the present case, the imbalance was temporal, stimulus durations for disk and ring being 18 and $100 \mathrm{msec}$, respectively. In addition, clearcut differences between the curves in overall likelihood of suppression are evident (Friedman two-way analysis of variance: $p<.001$ ), with green and yellow most readily detectable and

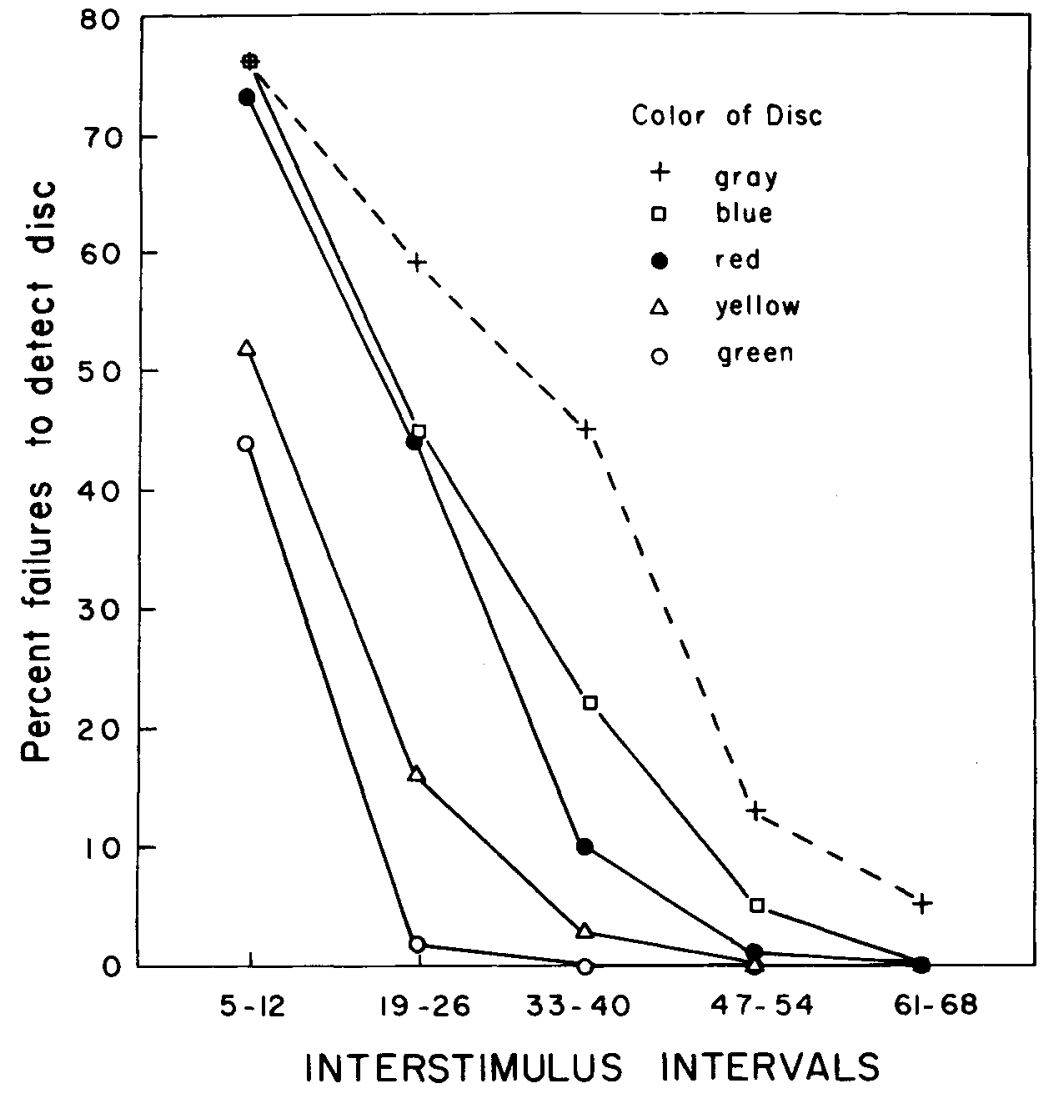

gray eliciting the greatest number of "No" responses. Differences between hues indicate that although matched on the Munsell value dimension, differences in detectability still remain, roughly reflecting the photopic visibility curve. In the case of the gray disk, it is possible that the viewing strategy adopted by $S$ could have produced an elevated number of "No" responses. S's task was primarily one of the discrimination of hues rather than the simple detection of stimuli. Thus, only when $S$ perceived no color did he have the secondary task of judging the brightness of the inner ring area-perhaps by matching it with the background-in order to determine whether it appeared gray. The demands of this second task thus may have produced a higher "threshold" for the gray disk.

\section{REFERENCES}

COX, S. I., DEMBER, W. N., \& SHERRICK, M. F. Effect on backward masking of spatial separation between target and mask contours and of target size. Psychonomic Science, 1969, $17,205-206$.
GRAHAM, C. H., \& BROWN, J. L. Color contrast and color appearances: Brightness constancy and color constancy. In C. H. Graham (Ed.), Vision and visual perception. New York: Wiley, 1965. Pp. 452-478.

KAHNEMAN, D. Method, findings, and theory in studies of visual masking. Psychological Bulletin, 1968, 70, 404-425.

KOLERS, P. A. Intensity and contour effects in visual masking. Vision Research, 1962, 2, 277-294.

KOLERS, P. A., \& ROSNER, B. S. On visual masking (metacontrast): Dichoptic observation. American Journal of Psychology, $1960,73,2-21$.

SCHILLER, P. H., \& SMITH, M. C. Detection in metacontrast. Joumal of Experimental Psychology, 1966, 71, 32-39.

WERNER, H. Studies on contour: I. Qualitative analyses. American Journal of Psychology, $1935,47,40-64$.

NOTE

1. This experiment was conducted under Contract N00014-67-A-0163-0001 between The Johns Hopkins University and the Physiological Psychology Branch, Office of Naval Research. 